
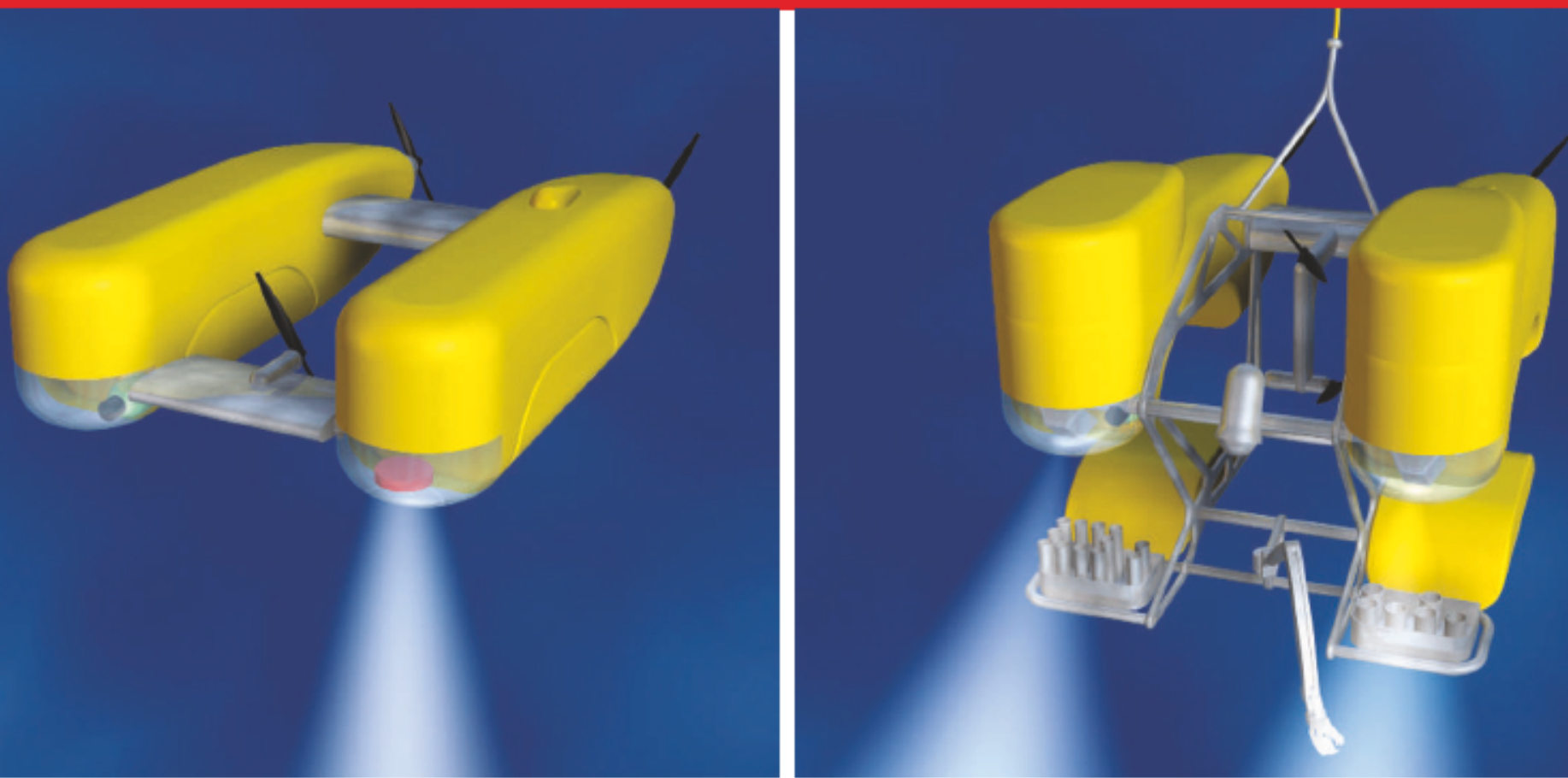

Freestyle: the HROV submersible will be rejigged from free-swimming mode (left) to tethered mode (right) aboard its mother ship.

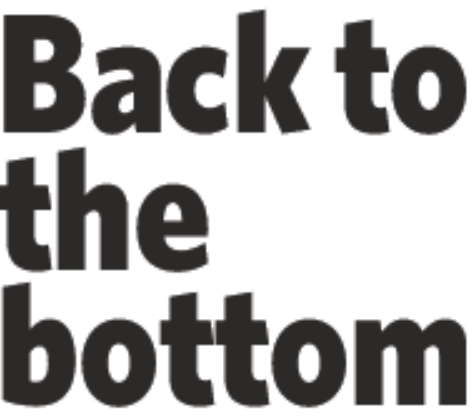

Marine scientists are getting ready for their newest tool, a versatile robot submersible that can travel into the oceans' deepest abyss. Robert Cooke visits the Massachusetts lab where the future of deep-sea exploration is taking shape.

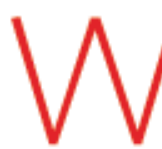
ith a typhoon bearing down, the operators of the ship Kairei made what seemed the sensible decision: they hauled in their lines and planned to leave the area. Only these were no ordinary fishing lines, but a kilometres-long stretch of cable leading to the world's deepest-diving submersible. And when Kaireis crew winched up the last of the cable, something was missing - the vehicle on the end, whose line had apparently snapped.

The future of deep-sea exploration darkened a bit on that stormy day in 2003 . The Kaireis submersible, Kaiko, was the star not only of the Japan Agency for Marine-Earth
Science and Technology but of the world's entire deep-diving fleet. In 1995, Kaiko had touched down on the bottom of the Challenger Deep in the Marianas Trench, 11,000 metres beneath the waves. It was only the second time a submersible had visited the legendary deep, and a first for a robotic craft.

But now, it looks as though Kaiko is to be replaced - and perhaps improved upon. At Woods Hole Oceanographic Institution (WHOI) in Massachusetts, home of the celebrated research submarine Alvin, marine engineers are preparing another vehicle to probe the deepest, darkest regions of the sea. Some components are still being designed, and even the overall shape has yet to be decided, but the engineers plan to have the vehicle in the water next summer and ready to dive to the Challenger Deep by 2007.

The craft, they say, will be much more than a replacement for the lost Kaiko or even for Alvin, which is due to be retired in the next four years or so. The new submersible will be a multi-talented, adaptable machine that can traverse the ocean in one of two configurations - self-guiding or controlled by surface operators through a whisker-thin fibre-optic cable. It's a combination of two successful marine exploration technologies - hence its unromantic designation of HROV, for Hybrid Remotely Operated Vehicle.

Like Alvin, but without a crew, the submersible will take pictures and video images, search with sonar signals, and grab rock and sediment samples off the sea floor. But on some missions, the HROV will also be sent off to go exploring on its own, in an autonomous mode for wide-area surveys.

Although the HROV will be the first submersible able to work either tethered or autonomously, the advantages of each mode are well proven. Autonomous underwater vehicles have made a name for themselves in 7 wide-field surveys and other tasks where con- 5 stant monitoring is not required (see Nature 421, 468-470; 2003). Freed from a tether, they can robotically 'fly' an underwater pattern to gather data on phenomena as different 8 as hydrothermal vents and algal blooms. क Remotely operated vehicles such as WHOI's 8 Jason II, on the other hand, have the advantage that they can be steered to particular spots of 8 interest, such as a newly formed hydrothermal $\frac{3}{3}$ vent. But the tethering cables produce drag, $\gamma$ making these vehicles harder and slower to 8 manoeuvre in the water.

\section{Control and freedom}

The \$5-million HROV is designed to have the best of both worlds. "We'll get a great deal of flexibility by being able to rearrange it," says Robert Detrick, vice-president for marine facilities and operations at WHOI.

In solo mode, the HROV will be programmed to follow instructions - to navigate through a search grid, for example - and then come back to its mother ship at the surface to deliver recorded data. If it locates something especially interesting, the HROV might be sent down again, tethered, to gather real-time data and respond minute-by-minute to commands from the surface. And it can do all that in a single day, with just one vehicle and one surface ship.

Better yet for marine scientists, the HROV will not be limited by depth. Alvin can safely reach 4,500 metres below the surface, which

\footnotetext{
"There is a very real risk. If one flotation ball implodes, the shock wave could take the whole vehicle out." - Andrew Bowen
} 
still leaves much of the ocean floor beyond its reach. The piloted Mir submersibles, owned by Russia, can reach 6,000 metres, as can the French submarine Nautile. Since the loss of Kaiko, Japan has continued to explore the depths using its manned Shinkai 6500 submersible, which can dive to 6,500 metres. The unmanned Jason II can also reach that depth.

Exploration of the deepest areas, however, must await the advent of the HROV. "The exciting thing is it should allow us to access areas that cannot be reached by existing manned vehicles," says Detrick. And there are discoveries to be made down there: when Kaiko visited the Challenger Deep, it brought back sediment containing primitive Foraminifera - single-celled organisms - that can withstand intense pressure. ${ }^{1}$ The only other craft ever to visit the deep was the French-built Trieste, which carried Swiss scientist Jacques Piccard and the US Navy's John Walsh to the bottom in 1960 .

But setting extreme-science records is not the HROV's goal, says Susan Humphris, a senior scientist at WHOI. Because of its multiple talents, the submersible will be useful at any depth. "One aspect I'm interested in is being able to access places we can't reach with current technology, especially in the Arctic," she says. Remotely operated vehicles generally aren't used in the Arctic for fear that ice might sever the tethering cables and the vehicle be lost. But with the HROV's dual capability, even if its cable is snapped it could still navigate itself back to its entry hole.

Because so little is known about the Arctic Ocean, any visit by the submersible is likely to yield important findings, says Humphris. She is particularly interested in scrutinizing the chemistry of Arctic seabed rocks: ${ }^{\alpha}$ These mantle rocks are the closest analogy we have today to what the whole planet's early materials were like", she says. Her group is already conducting preliminary experiments to test ideas about what geochemistry the HROV might find once it starts exploring beneath the ice.

Compared with other deep-diving submersibles, the HROV should be relatively easy to operate, in part because it won't need a dedicated ship and a large specialized crew to take it out to sea. Instead, all the necessary components will be in a van that can be lifted aboard almost any good-sized ship, with a small crew of dedicated technicians and engineers that will travel with it. The WHOI team aims to keep costs below $\$ 10,000$ per day, a third to a half as much as Jason II costs.

To keep costs low, much of the HROV technology will come off the shelf. The thin-walled ceramic spheres that will give the submersible buoyancy, for instance, are an offshoot of technology developed by the US beer company Coors, which worked on ceramics as a way to make better beer filters. And one key advance - the thin fibre-optic cable that links the HROV to the surface - was developed by the USNavy for torpedo guidance. The navyis

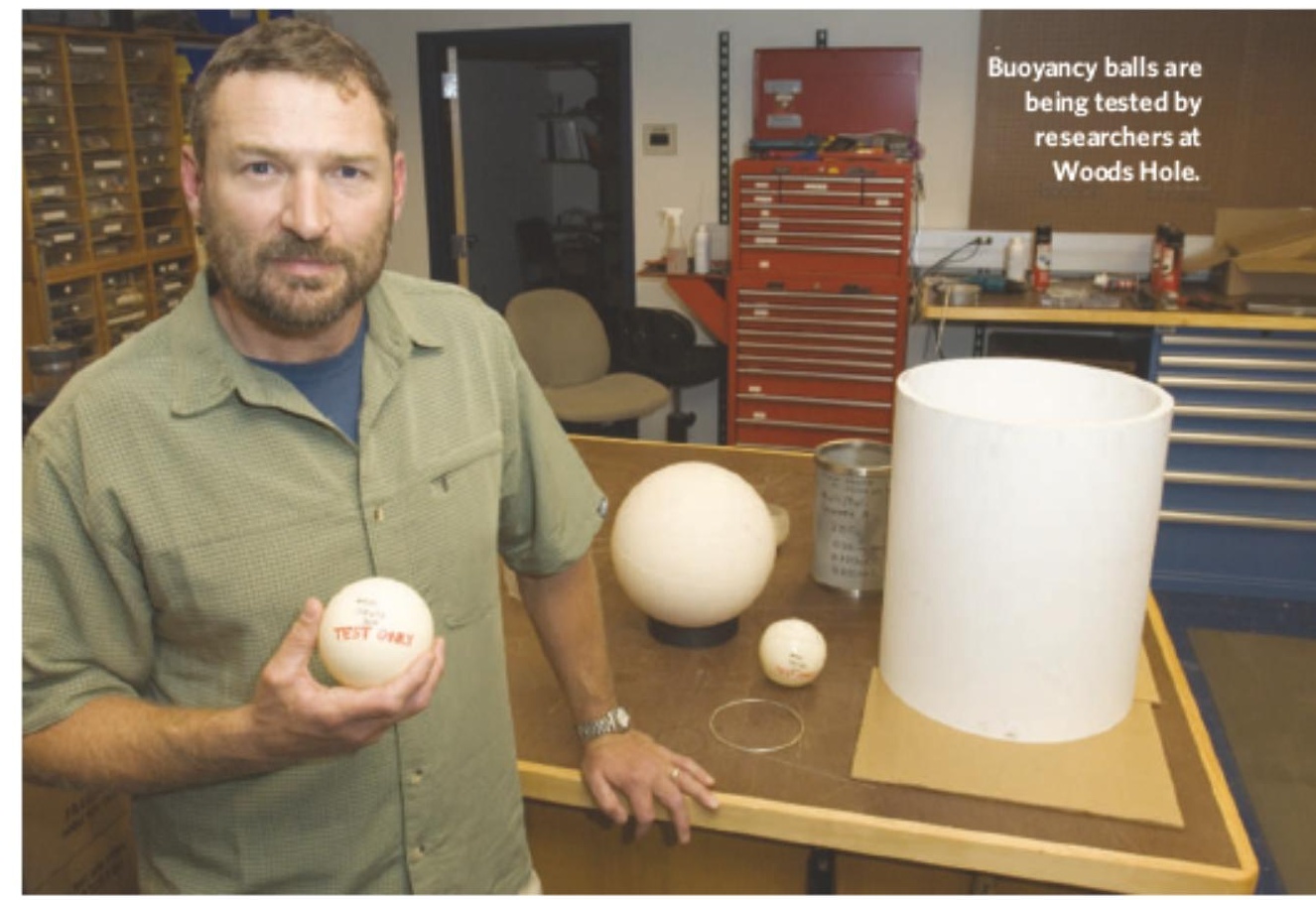

collaborating on the project, as is robotics expert Louis Whitcomb of Johns Hopkins University in Baltimore, Maryland.

\section{Batteries included}

Unlike Kaiko, which received electrical power through its tethering cable, the HROV will be powered by onboard batteries. Because the cable serves only as a communications link and not as a power cord, it can be very slender and far more lightweight than Kaiko's, for example. To make sure it won't snap, the WHOI team has already tested it by running a live link between a surface ship and the sea floor, says Andrew Bowen, leader of the engineering team.

Plans for the vehicle are coming together at

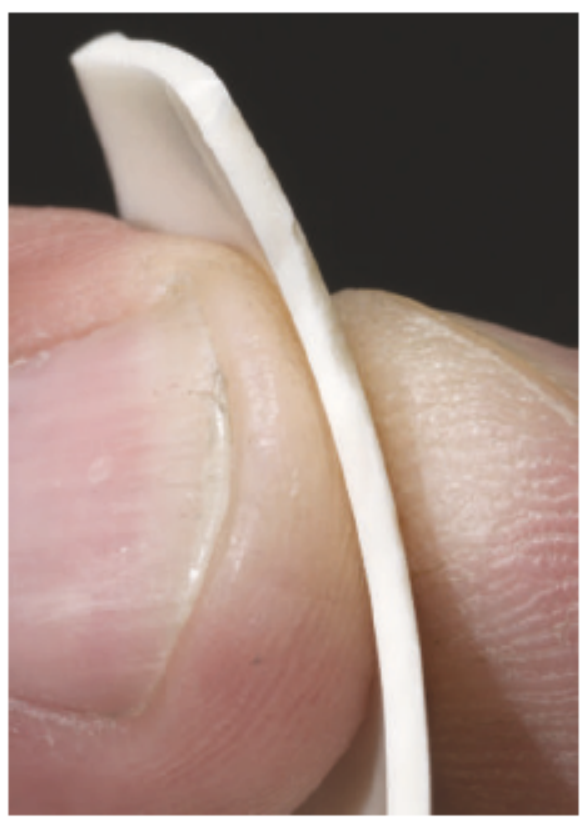

The thin ceramic shells of the buoyancy balls are tested to well beyond deep-ocean pressures.
WHOI's Deep Submergence Laboratory at the institution's picturesque campus on Cape Cod. Not far from the busy ferry terminal crowded with tourists, engineers cluster in a large, garage-like chamber. Tools, machinery and parts are scattered across the concrete floor. This is where the HROV will be built.

But first, the engineers have to decide which new technologies to include. ${ }^{2}$ "We're taking on the high-risk work early on," says Bowen. For instance, the team is experimenting with lowpower light-emitting diodes to illuminate the dark depths. They are also testing different ways of keeping the vehicle buoyant, such as the extraordinarily strong ceramic spheres that use the Coors technology.

In the lab, Bowen displays some examples, white balls from 9 to 20 centimetres in diameter. Engineers are trying to decide which size would be best. The spheres are being tested to withstand pressures of 200 megapascals, even though they're expected to encounter only 120 megapascals at the bottom of the Challenger Deep. Such rigorous testing is necessary, Bowen explains, because the HROV can't afford to have a flotation ball break under pressure at depth. "There is a very real risk," he says. "If one implodes, the shock wave could take the whole vehicle out." Ceramic enclosures - either spheres or cylinders - are also being considered to house the HROV's instruments, guidance and electronic systems.

Whatever it ends up looking like, the HROV will fill a prominent empty space in oceanography's tool-kit. Kaiko may be lost, but soon the HROV will take over its job exploring the deep sea floor.

Robert Cooke is a freelance writer in Stow, Massachusetts.

1 Todo, Y. etal. Science 307,689 (2005)

2. Bowen, A.D.etal.Max. Technol.Soc J.38, 92-101 (2004) 